$\mathrm{DOE} / \mathrm{ER} / 40233--8$

DE93 009370

\title{
TOPICS IN \\ GAUGE THEORIES AND THE UNIFICATION OF ELEMENTARY PARTICLE INTERACTIONS
}

\author{
Yogendra N. Srivastava \\ Michael T. Vaughn
}

Northeastern University

Boston, Massachusetts 02115

Progress Report

for period February 1, 1992 - January 31, 1993

February 1993

Prepared for

\author{
THE U.S. DEPARTMENT OF ENERGY \\ GRANT NO. DE-FG02-85-ER40233
}

\section{DISCLAIMER}

This report was prepared as an account of work sponsored by an agency of the United States Government. Neither the United States Government nor any agency thereof, nor any of their employees, makes any warranty, express or implied, or assumes any legal liability or responsibility for the accuracy, completeness, or usefulness of any information, apparrtus, product, or process disclosed, or represents that its use would not infringe privately owned rights. Reference herein to any specific commercial product, process, or seivice by trade name, trademark, ence herein to any sect necessarily constitute or imply its endorsement, recommendation, or favoring b e United States Government or any agency thereof. The views and opinions of authors expressed herein do not necessarily state or reflect those of the

United States Government or any agency thereof.

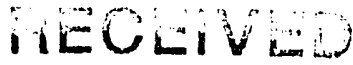 \\ MAR 221993 \\ ก.
}


Prógress Report on Grant DE-FG02-85ER40233

Period Covered: February 1, 1992 - January 31, 1993

\begin{abstract}
We report on work done by the principal investigators (Y. N. Srivastava and M. T. Vaughn) and their collaborators on

(1) Coulomb potential in G L ID on the lattice,

(2) Weyl cosmic strings and their consequences,

(3) interference between past and future events in $\phi \rightarrow K \bar{K}$,

(4) quantum effects of strong classical electromagnetic fields,

(5) application of computer algebra to calculations in supersymmetry and supergravity,

(6) numerical studies of classical scalar $\phi^{4}$ field theory and related nonlinear $\sigma$-models,

(7) analysis of a cubic model field theory, and

(8) renormalization group analysis of unified gauge theories.
\end{abstract}




\section{RESEARCH COMPLETED AND IN PROGRESS}

\section{Coulomb Potential in QED on the Lattice (Y. Srivastava and O. Panella)}

Approximating gauge field theories in continuous space-time by discrete lattice gauge models grew (in part) with the desire to compute numerically the consequences of quanturn gauge theories [1]. However, some features of the discrete lattice version of QED theory have been found more difficult than their counterparts in the continuous space-time theory. Such appears to be the case for the force law between charges in compact lattice QED [2].

For QED in continuous space-time, the law of force between two static charges is of course that of Coulomb, while the law of force between two steady state current distributions follows from Ampere's law. For lattice QED, the Ampere law can become somewhat difficult to compute due to the inherent nonlinear form of the field equations. On the other hand, the lattice analog of the Coulomb law can be directly computed. This lattice Coulomb law calculation was the purpose of the work.

The method used by us to compute rigorously the potential energy of a static charge distribution on the lattice fr, llows closely that of Weyl for ordinary QED in continuous space-time [3]. This energy is independent of temperature and can be calculated from the lattice version of Poisson's equation. It turns out to be the usual Coulomb potential. While this is not a startling result, its derivation starting from the the canonical Hamiltonian form [4] of compact QED on the lattice is interesting. It is described in ref. [5], and point to two unsolved problems:

(i) the imposition of the Gauss law constraint in lattice QCD through an appropriate Helmholtz theorem and

(ii) numerical computations of the radiation Hamiltonian in lattice QED.

References for Part 1.

1. K. Wilson, Phys. Rev. D10, 2445 (1974).

2. A. Polyakov, "Gauge Fields and Strings", Harwood Academic, New York (1987).

3. H. Weyl, "Group Theory and Quantum Mechanics", Dover Publications, New York (1950).

4. J. Kogut and L. Susskind, Phys. Rev. D9, 3501 (1974).

5. Y. Srivastava, O. Panella, M. Friedman and A. Widom, Phys. Lett. B 298, 405 (1993).

\section{Weyl Cosmic Strings and Their Consequences (Y. Srivastava)}

The notion of cosmic strings has been of importance in cosmology since the investigations of Kibble [1]. He noted that the condition for the existence of strings is that the unbroken symmetry subgroup of a unified gauge theory be disconnected when the the symmetry is spontaneously broken. Standard grand unified models, such as SUSY SU(5), SO(10), E6, ..., are thought to satisfy the Kibble criteria. However, for the considerations which follow, the microscopic details of the string core (apart from the mass per unit length) are not crucial. The length scale $L$ of grand unification and the mass density $\mu$ per unit length of string are related by

$$
\mu=\left(\hbar / 2 c L^{2}\right)
$$

The Vilenkin metric [2] for a cosmic string is that of "locally flat" space time

$$
c^{2} d \tau^{2}=c^{2} d t^{2}-d z^{2}-d \rho^{2}-\rho^{2} d \phi^{2},
$$


with (however) a small "angular deficit" $\beta,-\pi+\beta<\phi<\pi-\beta$ with

$$
\beta=\left(2 G \mu / c^{2}\right)=(\Lambda / L)^{2}
$$

In Eq. (3), $\Lambda$ is the Planck length. The Vilenkin metric implies (due to local flatness) that a test mass placed at rest near the string would not be accelerated toward the string. (This conclusion was modified in more iecent work in which a small gravitational mass is induced by vibrations in the string.) It is of interest to compare the Vilenkin string with the Newtonian string.

In Newtonian gravitational theory, a string with mass density $\mu$ per unit length produces a gravitational potential

$$
\Phi(\rho)=(2 G \mu) \ln (\rho / L)
$$

and thereby a gravitational acceleration

$$
g=-(d \Phi(\rho) / d \rho)=-(2 G \mu / \rho)=-\left(\beta c^{2} / \rho\right)
$$

Thus a test particle moving in a circular orbit about a Newtonian string with velocity $v_{\infty}$ accelerates at $g=-\left(v_{\infty}^{2} / \rho\right)$ which together with Eq. (5) implies that

$$
v_{\infty}=c \sqrt{\beta}=c(\Lambda / L)
$$

Taking the ratio of the Planck length to the unification length

$$
(\Lambda / L) \sim 10^{-3}
$$

as suggested by the LEP data [3], yields

$$
v_{\infty} \sim 300 \mathrm{~km} / \mathrm{sec}
$$

which is roughly the rotational velocity of stars near the outside of galaxies [4].

This relationship between grand unification length scales (coupling $\beta \sim 10^{-6}$ ) and the asymptotic rotational velocities of stars in many galaxies suggests the following hypothesis: Galaxies are localized about cosmic strings due to the gravitational attraction between visible galactic matter and the string mass density. The asymptotic velocity $v_{\infty}$ is related to the string mass density $\mu$ as in Eqs. (6)-(8). No recourse is made to the notion of "dark matter" producing $v_{\infty}$ beyond the string mass density $\mu$. (Many schemes for galaxy formation require both strings and dark matter. The above renders the dark matter superfluous.) One consequence of this hypothesis is that galaxies should have a filamentary distribution. There is a considerable literature on this subject, but the final results indicate that filamentary distributions are very common [5].

Of course the idea of a Newtonian string is simple but would not be of much theoretical importance were there not a general relativistic metric with an appropriate Newtonian limit. Let us here discuss the metric of interest. The Weyl cosmic string can be described by the metric [6]

$$
c^{2} d \tau^{2}=(\rho / L)^{2 \beta} c^{2} d t^{2}-(L / \rho)^{2 \beta} \rho^{2} d \phi^{2}-(L / \rho)^{2 \beta(1-\beta)}\left(d \rho^{2}+d z^{2}\right)
$$

To see how the Weyl string of Eq. (9) is related to the Newtonian string of Eqs. (4) and (5) we note that 
the energy of a test mass $m$ at rest in the Weyl matric is given by

$$
U=m c^{2}(\rho / L)^{\beta}
$$

so that the weight of the test mass due to the attraction of the string is given by

$$
W=-(d U / d \rho)=-\left(\beta m c^{2} / \rho\right)(\rho / L)^{\beta}=m g(\rho / L)^{\beta},
$$

where $g$ is the gravitational acceleration calculated on the basis of Newtonian potential theory and

$$
(\rho / L)^{\beta}=e^{\beta \ln (\rho / L)}
$$

describes the correction due to general relativity. Even though the ratio of the galactic radius to the grand unification length $(\rho / L)$ is truly enormous, the weak coupling $\left(\beta \sim 10^{-6}\right)$ still insures that the factor in Eq. (11) giving the correction to the Newtonian string from general relativity is very close to unity.

The Weyl string will bend light much as the Schwarzschild metric yields the bending of light around a central mass (e.g. the sun). For a light ray directed normal to the string axis, the change in the scattering angle is given (to a sufficient degree of accuracy) by

$$
\Delta \Theta=2 \pi \beta+\ldots
$$

in qualitative agreement with the Vilenkin string angular deficit in Eq. (3). However, although the Weyl string bends light, there is no angular deficit.

In contrast to massless particles (such as the photon) which do scatter and hence escape away from the string, massive particles always remain bound to the string. Because the string's cross section for scattering photons is so small, optical detection would be difficult but perhaps not impossible. Both the AharonovBohm type phase shift and gravitational lensing, predicted for Vilenkin strings [2], would also occur in the Weyl case.

As stated above, massive particles are actually bound to the string by an approximately logarithmic potential. Therefore, it is in principle possible to detect Weyl strings by their effects on the interstellar medium. Specifically, particles in planar orbits around a string would begin to move helically down the string's length when subjected to a perturbation in the axial direction. This effect could result in bulk flows of interstellar matter along well defined, comparatively narrow paths between galaxies threaded by a string. In fact, more that twenty such intergalactic "bridges" or "tails" have been observed [7], the most famous being the Magellanic Stream in our own local group [8].

It is also interesting to note, that in recent years over one hundred small, mostiy parallel, linear features have been discovered inside the Milky Way, aligned with and concentrated near the galactic axis [9]. One such structure (a thirty parsec "thread-like" gas arc and "double jet" parallel to the galactic axis) is situated at Sagittarius A, apparently at the exact center of the galaxy [10].

Finally, the recent COBE experiment [11] on the background cosmic radiation temperature ainisotropy is consistent with the estimate $(\Delta T / T) \sim \beta$ which follows from the scattering of radiation from Weyl strings.

This work has been submitted for publication [12]. 
References for Part 2.

1. T. W. B. Kibble, J. Phys. A9, 1387 (1976); "Cosmic Strings - an Overview" in "The Formation and Evolution of Cosmic Strings", Cambridge University Press, Cambridge (1990).

2. A. Vilenkin, Phys. Rev. 24D, 2082 (1981); A. Vilenkin, in "300 Years of Gravitation", p. 499 (S. Hawking and W. Israel, eds.), Cambridge University Press, Cambridge (1987); B. Carter, Phys. Rev. 41D, 3869 (1990).

3. U. Amaldi, W. De Boer, and H. Furstenau, Phys. Lett. B260, 447 (1991).

4. M. V. Zombeck, "Handbook of Space Astronomy and Astrophysics", p. 22, Cambridge University Press, Cambridge (1982).

5. P. Argyres et al., Astronomical Journal 91, 471 (1986); B. Binggeli, Astronomy and Astrophysics 107, 338 (1982); H. Muriel and D. G. Lambas, Astronomical Journal 98, 1995 (1989).

6. H. Weyl, Ann. Phys. (Leipzig) 54, 117 (1917); C. Reina and A. Treves, Gen. Rel. and Grav. 7, 817 (1976).

7. M. P. Haynes, Astronomical Journal, 86,1126(1981). M. S. Roberts, "New Ideas In Astronomy", p. 72, (F. Bertola, ed.), Cambridge University Press, Cambridge (1988).

8. D. S. Mathewson et al., Astrophysical Journal 190, 291 (1974).

9. C .Heiles et al., Astrophysical Journal 242,533 (1980); B-C. Koo et al., in "The Interstellar Disc-Halo Connection in Galaxies", p. 65, (H .Bloemen ed.), Kluwer Academic Press, Dordrecht (1991).

10. M. Morris and F. Yusef-Zadeh, Astronomical Journal 90, 2511 (1985); F. Yusef-Zadeh et al., Astrophysical Journal 300, L47 (1986).

11. G. Smoot "Cosmic Microwave Background Anisotropy Detected by the COBE DMR", Invited talk at the International Conference on High Energy Physics, Dallas, Aug. 1992.

12. Y. Srivastava, A. Widom and N. Redington, submitted to Phys. Rev. D.

\section{Interference Between Past and Future Events in $\phi \rightarrow K \bar{K}$ (Y. Srivastava)}

In a remarkable paper [1], Einstein, Tolman and Podolsky discussed a rather "counter-intuitive" result in quantum mechanics, namely, that uncertainties in the "future" events can be reflected in the probability distributions of "present experiments". We have put the ETP observation mathematically in the form of dynamic uncertainties at different times. For any two hermitian operators $A\left(t_{1}\right)$ and $B\left(t_{2}\right)$, consider the time-ordered Feynman Green's function

$$
G_{A B}\left(t_{1}, t_{2}\right)=(i / \hbar)<T\left\{A\left(t_{1}\right) B\left(t_{2}\right)\right\}>
$$

Using the Weyl inequality, it is then easy to show that

$$
\Delta A\left(t_{1}\right) \Delta B\left(t_{2}\right) \geq \hbar\left|\operatorname{Re} G_{A B}\left(t_{1}, t_{2}\right)\right| .
$$

The central point of the ETP effect is that uncertainties in physical quantities at two different times must be viewed in a time symmetric manner. Past measured uncertainties will be reflected in future (to be measured) uncertainties - which is all quite reasonable. That future measured uncertainties will be reflected in past measured uncertainties is less intuitive, but is also a consequence of quantum mechanics as described by ETP. 
We have provided a concrete proposal for an experimental test of this notion through $\phi$ factories such as . the DA $\Phi$ NE project at Frascati $[2,3]$. The central idea is to observe amplitude interference between future and past events through the time-reversal violating decays of the neutral $K \bar{K}$ puirs.

Several groups (see references $[2,3]$ for a detailed bibliography) have derived that the transition rate for $K$-decay channel $c$ in time $d t_{c}$ and $K$-decay channel $d t_{d}$ is given by

$$
d^{2} P_{c d}\left(t_{c}, t_{d}\right)=\left|\Psi_{c d}\left(t_{c}, t_{d}\right)\right|^{2} d t_{c} d t_{d}
$$

where the "two-time" amplitude

$$
\Psi_{c d}\left(t_{c}, t_{d}\right)=\psi_{L}^{c}\left(t_{c}\right) \psi_{S}^{d}\left(t_{d}\right)-\psi_{L}^{d}\left(t_{d}\right) \psi_{S}^{c}\left(t_{c}\right)
$$

and the single decay probability amplitudes are given by

$$
\psi_{k}^{a}\left(t_{a}\right)=A_{k}^{a} \exp \left[-i\left(m_{k} t_{a}\right)-\left(t_{a} / 2\right)\right]
$$

To probe whether and how a $K$-meson carries information about its future environment, consider the situation showed in Fig. 1. The $\phi$ decays at time zero into two uncharged $K$ mesorss, one of which decays $K \rightarrow \pi^{\circ} \pi^{\circ}$ at time $t$ and the other which decays at a later time $T$, induced to do so by a condensed matter absorbing bar. From the point of view of Feynman diagrams, the interfering amplitudes do exist from future to past events. This implies that the probability of the earlier $K \rightarrow \pi^{\circ} \pi^{\circ}$ event ought to depend upon where ad when the other $K$ meson hit at a later time $T$ the absorbing bar.

To make the above statements quantitative, we show in Fig. 2 the number of interfering events $\Delta N$ over the total number $N$ of events of decays up to a given time $3 \tau_{s}$, calculated as a function of $T$ for an ideal absorber. For the velocities under consideration, all $T \geq \frac{9}{2} \tau_{s}$ are in the future light cone of the earlier decay. For over $10^{9} \mathrm{KK}$ pairs per year planned at $\mathrm{DA} \Phi \mathrm{NE}$, the effect appears measurable. Moreover, any experimental determination of the ratio $\epsilon^{\prime} / \epsilon$ would require subtractions of the ETP background.

We are presently investigating alternative setups using regeneration type exjeriments for further probing of this important effect at yet larger times.

References for Part 3.

1. A. Einstein, R. C. Tolman and B. Podolsky, Phys. Rev. 37, 780 (1931).

2. G. Pancheri, ed., "Workshop on Physics and Detectors for DAФNE", LNF Report Frascati (1991).

3. L. Maiani, G. Pancheri and N. Paver, eds., The DAФNE Physics Handbook (1992), LNF Report Volume I, Frascati (1992).

4. Y. Srivastava and A. Widom, Laboratori Nazionali di Frascati, INFN, LNF Preprint, February 1993. 


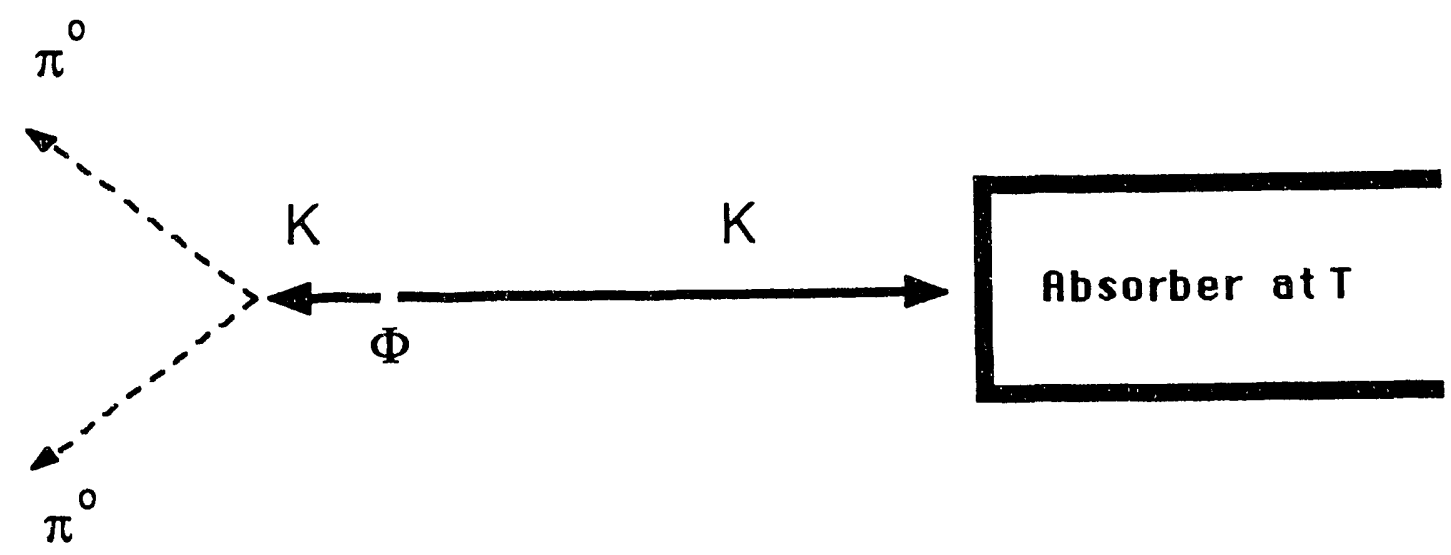

Fig. 1. Schematic setup to observe the ETP effect in the decay $\phi \rightarrow K \bar{K}$.

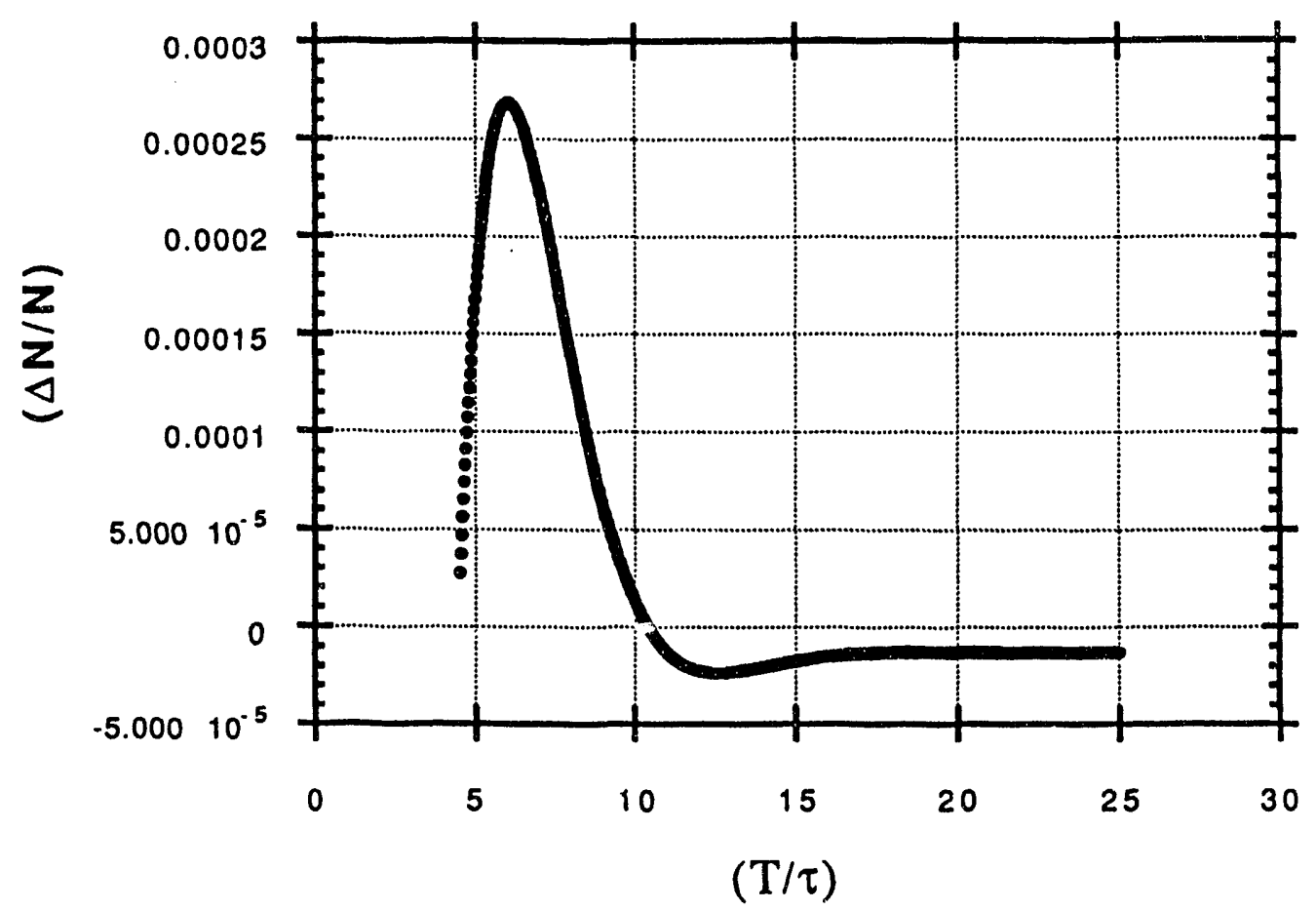

Fig. 2. The fractional number $\Delta N / N$ of interfering events for $K$ decays up to time $3 \tau_{s}$, calculated as a function of the later time $T$ at which the other $K$ reaches an ideal absorber. 
4. Quantum Effects of Strong Classical Electromagnetic Fields (E. Sassaroli and Y. Srivastava)

This is a program to systematically study quantum effects of strong classical electromagnetic fields. To this point, there have been three major studies:

(i) stimulated photon emission In QED [1],

(ii) electron-positron production in a strong electromagnetic field [2],

(iii) resonance structures in $e^{+}-e^{-}$pair production in time-dependent electromagnetic fields [3].

In the first paper (on stimulated photon emission In QED [1]), it is shown how the dynamical Casimir effect can give origin to the " $1 / \omega "$ noise in electronic devices. That is, if the electromagnetic vacuum is modulated by a pulse in time this appears in laboratory situations as an excess noise after the pulse and the spectrum shows a $1 / \omega$ behavior as the frequency $\omega$ approaches zero.

In the second paper, the $\mathrm{e}^{+} \mathrm{e}^{-}$pair production probability in a strong electromagnetic field is calculated using a semi-classical method. The possibility of creating particle pairs by highest power lasers presently available is also discussed.

In the third paper, electron-positron pair production in time dependent potentials is considered. The pair production probability as a function of the energy has been calculated and it shows a resonance structure. Some numerical applications to heavy atom collisions are also described.

References for Part 4.

1. A. Widom, Y. Srivastava and E. Sassaroli, "Dynamical Casimir Effect and (1/ $\omega)$-noise", Invited Talk by Y. Srivastava at the Chia Conference on the Interface Between Condensed Matter and High Energy Physics, September 1992 (to appear in Nuclear Physics).

2. E. Sassaroli, Y. Srivastava and A. Widom, "Electron-Positron Pair Production in a Strong Electromagnetic Field", submitted for publication to Nuovo Cimento A.

3. E. Sassaroli, Y. Srivastava and A. Widom, "Resonance Structures through Time-Dependent Potentials", manuscript in preparation. to Nuovo Cimento A.

\section{Application of Computer Algebra to Calculations in Supersymmetry and Supergravity (Y. Srivastava and V. Lucic)}

Analytic calculations in different supersymmetry (SUSY) and supergravity (SUGRA) theories tend to be very tedious; the equations are in general complicated and have many terms. Nevertheless, most of the rules used in the calculations are rather clear and their application straightforward. Thus these equations are prime candidates for using various symbolic manipulation computer programs. Unfortunately, none of the programs are developed specifically for such calculation. Therefore, in order to deal with SUSY and SUGRA, we must develop routines for calculations using Grassman (noncommuting) variables and to include operations on spinors, or spinor-tensors in general. Our efforts were concentrated on two problems which follow.

It was natural to begin the work on $N=1$ SUSY theory in four space-time dimensions. This part was done using Reduce symbolic manipulation program, following conventions introduced by Wess and Bagger [1]. Although the results obtained are correct and absolutely satisfying, it turns out that it is very difficult to generalize the procedure in Reduce to use gauge fields, for instance. For that reason we decided to 
proceed with Mathematica [2] software. It has more capabilities than Reduce, but some basic things, such as anticommutation rules, are much more difficult to program. Unfortunately, the two programs are so different that everything has to be redone from the beginning.

The problem we attacked is related to the suoergravity theory of Friedman and Srivastava [3]. The equations of motion of the theory are expressed in terms of a curvature, which is a superfield (defined on a space having both Bose and Grassman coordinates). In addition, the curvature has indices, both Bose and Grassman, in a representation of super-Poincare group. The problem was to express the equations of the motions in terms of curvature fields. Since the initial equation of motion had Grassman derivatives, these operation had to be defined and performed, as well as the operation of the ordinary derivatives, since the Mathematica rules for them were unsatisfactory. As the last step, terms with the same Grassman coordinate dependence were extracted from the each of the equations. For a two Grassman coordinates, $N=1$ case, $8 \times 4=32$ equations were thus obtained, each in terms of connection fields, as required.

References for Part 5.

1. J. Wess and J. Bagger, "Supersymmetry and Supergravity", Princeton University Press (1983).

2. S. Wolfram, "Mathematica: a system for doing mathematics by computer", Addison-Wesley (1988).

3. M. Friedman and Y. Srivastava, Phys. Fev. D15, 1026 (i977); Phys. Rev. D16, 304 (1977); Phys. Rev. D18, 4387 (1978).

\section{Analysis of Classical Scalar $\phi^{4}$ Field Theory (M. Vaughn)}

From various analyses which have been carnied out [1-8], it seems likely that high-energy scattering leading to large numbers of bosons in the final state is not amenable to an ordinary perturbative treatment. T hus we (H. Goldberg, D. Nash, and myself) were led to a study of the classical $\lambda \phi^{4}$ theory. In particular, we wanted to study of the flow of energy in the classical system between normal modes of the free field, starting from initial conditions in which the energy resides in a few high-frequency modes of the free field (somewhat snalogous to a field configuration which might be produced in a high-energy collision). We then followed the energy in the various modes of the field as a function of time, to see if it is dissipated into many 'quanta' of the low-frequency modes.

We did extensive numerical studies on both the $(1+1)$-dimensional theory and a special class of spherically symmetric solutions in the $(3+1)$-dimensional theory (with $m^{2}>0$ ), and found some rather striking behavior, which is rather puzzling to us, and no sign of cascading of the energy into low-frequency modes. For weak coupling, the energy tends to stay in the initial modes, with some excursion into nearby modes; we can understand this as a motion of the field in quasi-normal modes which have been slightly distorted from the normal modes of the free field by the interaction. As the coupling strength is increased, the energy disperses more widely, but nevertheless rather slowly on a scale of the frequency of the field as a whole (since we are confining the field to a finite region of size $L$, the lowest natural frequency of the field is $\omega_{0} \sim \sqrt{(\pi / L)^{2}+m^{2}}$ with corresponding time scale $\left.T \sim 2 \pi / \omega_{0}\right)$. In the $(1+1)$-dimensional theory, there is some evidence for quasi-periodic motion, though not a full return to the initial modes, over tim. spans $\sim 1000-2000 T$, up to relatively large coupling constants.

Two striking features of the results in the $(3+1)$-dimensional theory can be nnted:

(i) The tim: for the energy in the initial modss to decrease to $\sim 40 \%$ of its initial value is strictly proportional to $1 / \lambda$ over a wide range of $\lambda$ (almost two orders of magnitude), although for very weak coupling, the energy in the initial modes never falls that low before starting to return to the initial modes. 
(ii) If we define the number of 'quanta' $J_{n}$ in the $n^{\text {th }}$ normal mode as $E_{n} / \omega_{n}$, where $E_{n}$ is the energy, and - $\omega_{n}$ the frequency of the $n^{t h}$ normal mode, the the total number of 'quanta' $N \equiv \sum_{\text {modes }} J_{n}$ is approximately conserved over times as long as $5000 T$, even when the coupling constant $\lambda / 4 \pi \sim 1.0$. This number is not exactly conserved - it fluctuates over short time scales, but by much less than a simulated distribution obtained by distributing energy randomly among a relatively narrow band of modes. This indicates the existence of some integrability in the classical system beyond conservation of energy.

It turns out that this near-conservation law can be at least partly understood in classical perturbation theory. If we formulate the theory in terms of the normal modes of the non-interacting theory, and transform the classical Hamiltonian to the action-angle variables associated with these modes, then the number of 'quanta' in a normal mode can be identified with the corresponding action variables. When the $\lambda \phi^{4}$ interaction is expressed in terms of the action-angle variables, there are terms which conserve the total number of 'quanta', and terms which do not. However, classical analysis shows that only the terms which conserve 'quanta' contribute to the secular transfer of energy between modes - the other terms only give rise to small-amplitude high-frequency oscillations in the number of 'quanta' (which we have also observed). 'This result is due to the mass of the fields and the fact that we have chosen modes which are relativirtic, but not extremely so. Hence the beat frequencies, which measure how far the process is off the energy shell in the quantum system, are smaller for the transitions which conserve quanta than for those which do not.

We note that the recurrent behavior in a system of coupled oscillator first discovered numerically by Fermi, Pasta and Ulam [9] is not relevant here - that system is known to reduce to a completely integrable system in the continuum limit, while the $\lambda \phi^{4}$ theory (with $m^{2}>0$ ) has no such rich structure. The standard works [10-12] on classical systems with few degrees of freedom provide us with some ideas, but no results directly applicable to the field theoretic system. While the applicability of our results to the quantum problem is unclear at the present, we believe the results are of intrinsic interest in the classical context.

Our results on the $(3+1)$-dimensional system have been published [13]. W's have further results on the $(1+1)$-dimensional theory, which are similar to those in the $(3+1)$-dimensional case. We are trying to decide whether the system warrants a more detailed study in which the behavior of the system is traced as a function of coupling constant to see if there is some kind of 'phase transition' from the perturbative behavior to chaos.

References for part 6.

1. A. Ringwald, Nucl.Phys. 330, 1 (1989).

2. O. Espinosa, Nucl.Phys. 343, 310 (1990).

3. J. M. Cornwall, Phys. Lett.243, 271 (1990).

4. H. Goldberg, Phys. Lett.246, 445 (1990) .

5. M. B. Voloshin, TPI preprint TPI-MINN-90/54-T (1990).

6. L. N. Lipatov, Sov. Phys. JETP 45 (2), 216 (1977).

7. E. Brézin, J.C. Le Guillou, and J. Zinn-Justin, Phys. Rev. D15, 1544 (1977).

8. H. Goldberg and M. T. Vaughn, Phys. Rev. Letters 66, 1267 (1991).

9. E. Fermi, J. Pasta and S. Ulam, Studies of Non-linear Problems, Los Alamos report LA-1940 (1955), reprinted in Collected Works of Enrico Fermi, vol. II, p. 978, University of Chicago Press (Chicago, 1965).

10. V. I. Arnold, Mathematical Methods of Classical Mechanics, Springer-Verlag (New York, 1978). 
,

11. A. J. Lichtenberg and M. A. Lieberman, Regular and Stochastic Motion, Springer-Yerlag (New York, 1983).

12. M. C. Gutzwiller, Chaos in Classical and Quantum Mechanics, Springer-Verlag (New York, 1990).

13. H. Goldberg, D. Nash and M. T. Vaughn, Phys. Rev. D46, 2585 (1992).

\section{Analysis of a Cubic Model Field Theory (M. Vaughn)}

Further insight into the behavior of miltiparticle amplitudes for large $N$ can be found in a 'cubic' model [1] defined by the Hamiltonian

$$
\mathcal{H}=M \sum_{p=1}^{\infty} a_{p}^{\dagger} a_{p}+\frac{1}{2} \lambda \sum_{l, m=1}^{\infty}\left(a_{l+m}^{\dagger} a_{l} a_{m}+a_{l}^{\dagger} a_{m}^{\dagger} a_{l+m}\right)
$$

where $a_{p}$ and $a_{j}^{\dagger}$ are creation operators for a particle with an $i c$ quantum number $p$, which we can call 'momentum'. The operator corresponding to this quaritum number is

$$
\mathcal{P} \equiv \sum_{p=1}^{\infty} p a_{p}^{\dagger} a_{p}
$$

Since $\mathcal{P}$ commutes with the Hamiltonian (3), the system separates into sectors characterized by integer eigenvalues of $\mathcal{P}$. Thus, the Hilbert space factorizes into subspaces with definite $P$. Because of the positivity of all of the momenta, these will be finite dimensional subspaces.

To simplify our analysis, we also restricted ourselves to states in which no more than one particle with a particular momentum $p$ is present. Thus, we exclude 'laser' effects, with their attendant $\sqrt{n_{i} !}$ factors in matrix elements. In this sense, these are "hard core" bosons: there is a fermionic exclusion principle without explicit anticommutation relations and antisymmetrization.

To study amplitudes associated with transitions to and from an $N$-particle state, we can conveniently think in terms of a maximal state, namely an $N$-particle state with momenta $k=1,2, \ldots N$. This state will have a total momentum $P=N(N+1) / 2$. We will consider only such sectors, labeled by $N$, which have this maximal state in their spectrum.

In general, the total number of states $\mathcal{N}_{P}$ for a given $P$ is given by the exponent of $x^{P}$ in the expansion of the generating function

$$
\prod_{j=1}^{\infty}\left(1+x^{j}\right)=\sum_{P=0}^{\infty} \mathcal{N}_{P} x^{P}
$$

If we consider the states coupled to a maximal state with $N=2,4,8,16 \ldots$ (corresponding to $P=$ $3,10,36,136 \ldots$ ), we find that the number of states to be considered is $2,10,668,7215644, \ldots$, respectively; the number of states increas dramatically with increasing $N$. Even going from $N=8$ to $N=9$ means increasing the subspace from 668 to 2048 states. Thus, in a practical decision based on CPU time considerations, we restricted our numerical analysis to the case $N=8$.

We find (1) that there is reasonable period of time for which there is a cunstant rate for the $1 \Rightarrow 8$ transition; (2) at the end of the linear period, the eight particle amplitude attains a maximum value $\left|A_{8}\right|_{\max }$ which is about $3-4$ orders of magnitude larger than the comparable amplitude for excitation of the $N=8$ 
state in the anharmoric oscillator in a model introduced by Bachas [2], and (3) for values of the coupling in the region where the Born approximation fails, the amplitude is much larger than the naive estimates $A_{8} \simeq \exp \left(-1 / g^{2}\right)$ or $\exp (-8)$; it is more like $A_{8} \sim \exp \left(-0.20 / g^{2}\right)$. Further analysis of the model by Goldberg [3] suggests that this weakening of the exponential suppression of the amplitude persists for larger $N$.

References for part 7 .

1. H. Goldberg and M. T. Vaughn, Phys. Lett. B291, 125 (1992).

2. C. Bachas, École Polytechnique preprint A089.1191 (December 1991).

3. H. Goldberg, Northeastern University prcprint (April 1992)

\section{Renormalization Group Analysis of Unified Gauge Theories (M. Vaughn)}

There are two general areas of renormalization group (RG) analysis which are of great current interest:

(i) the use of RG analysis to constrain or predict particle masses, and

(ii) extc...in of RG analysis beyond one loop, with special attention to the transition between different efft. heories as energy scales are varied.

In the standard calculations of the two-loop beta-functions [1-4], only the dimensionless coupiing constants of a general field theory were considered, although the work of Jack and various collaborators $[3,4]$ has consiçred the effective potential in various models.

In collaboration with Steve Martin (a post-doc at Northeastern), I have started a calculation of the general two-loop evolution of scalar masses and the (dimensional) coupling constants which can occur in the self-interaction of scalar fields. These cubic couplings do not occur in the unbroken minimal standard model, but they do occur naturally in supersymmetric extensions, in which there are couplings of the type squark-squark-Higgs and slepton-slepton-Higgs (squark and slepton are the scalar supersymmetric partners of the standard model quarks and leptons).

In the minimal supersymmetric standard model with "universal" (flavor-independent) soft supersymmetry breaking terms, it is possible to make some predictions regarding the mass spectrum of the squarks and sleptons in terms of a few unknown parameters. This involves renormalization group running of the squark and slepton mass parameters from a very high energy scale (where they are assumed to be universal) down to the electroweak scale. A number of authors have performed such analyses using the one-loop renormalization group equations [5-7].

If the idea of supersymmetry proves to be correct, it will be very exciting to experimentally test the spectrum of squarks and sleptons which emerges in this way. In order to achieve greater accuracy in predicting the features of the spectrum of squarks and sleptons, it will be very useful to have general expressions for the two-loop renormalization of scalar masses. In many regions of parameter space, the effect of the two-loop contributions on the predictions for scalar masses can be quite appreciable. Therefore we propose to calculate the renormalization group running of scalar masses; first in a general renormalizable theory containing scalars, fermions, and gauge fields, and then specializing to the case of the minimal supersymmetric standard model with general soft supersymmetry breaking terms. Some work on two-loop revolution has already been done [8-10], but only for specific models.

We also hope to be able to say something about the scheme dependence (dimensional regularization vs. dimensional reduction) in the SUSY models, which was left partly unresolved in 1984 [1-3]. 


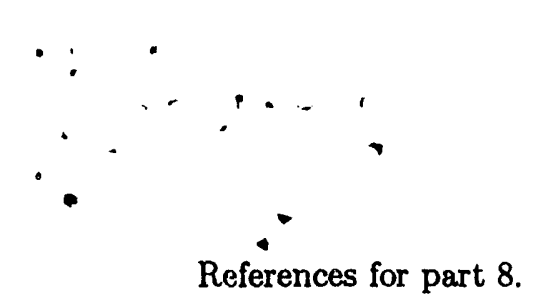

1. M. T. Vaughn and M. E. Machacek: (a) Nucl. Phys.B222, 83 (1983); (b) B236, 221 (1984); (c) B249, 83 (1985).

2. M. Fischler and J. Oliensis, Phys. Lett. 119B, 385 (1982); Phys. Rev. D28, 2027 (1983); R. van Damme, Nucl. Phys. B227, 317 (1983); B244, 105 (1984).

3. I. Jack, J. Phys. A16, 1083 (1983); I. Jack and H. Osborn, J. Phys. A16, 1101 (1983); Nucl. Phys. B249, 472 (1985).

4. A recent paper by C. Ford, I. Jack and D. R. T. Jones, Nucl.Phys.B387 373 (1992), discusses the two-loop effective potential in the standard model, and corrects two minor errors in ref. 1 (c).

5. K. Inoue, A. Kakuto, H. Komatsu, S. Takeshita, Prog. Theor. Phys. 68, 927, (1982).

6. L. Alvarez-Gaumé, J. Polchinski and M. Wise, Nuci. Phys. B221, 495, (1983).

7. L. E. Ibáñez, Nucl. Phys. B218, 514 (1983); L. E. Ibáñez and C. López, Phys. Lett. 126B, 54, (1983); Nucl. Phys. B233, 511, (1984)

8. J. R. Espinosa and M. Quiros, Phys. Lett. B266, 389 (1991) have calculated the two-loop corrections to the mass of the lightest Higgs scalar in SUSY models.

9. H. Arason, D. J. Castano, B. Keszthelyi, S. Mikaelian, E. J. Piard, P. Ramond and B. D. Wi ight, Phys. Rev. Lett. 67, 2933 (1991) and Phys. Rev. D46, 3945 (1992) have looked at the two loop constraints on masses in both SUSY and non-SUSY models.

10. V. Barger, M .S. Berger and P. Ohmann, University of Wisconsin prepriuts (Sept. 1992 and Oct. 1992) have looked at the evolution of masses and also of the CKM matrix elements at two loops. 


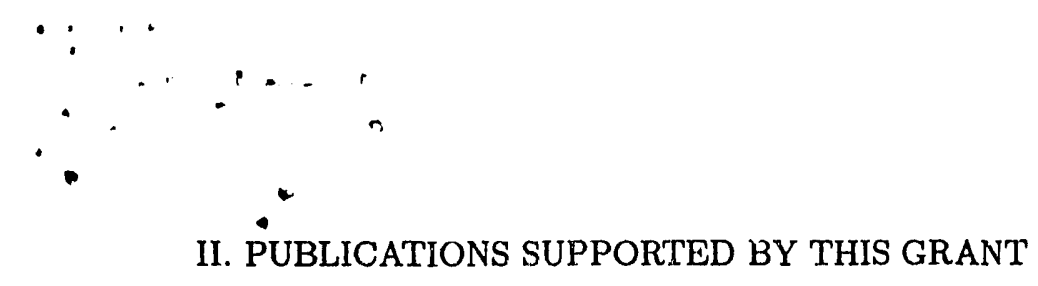

1. Y. Srivastava and A. Widom, "Gravitational Diamagnetism", Phys. Lett. B 280, 52 (1992).

2. A. Widom, Y. Srivastava and $N$, Redington, "Weyl Cosmic Strings and their Consequences", submitted for publication to the Physical (1. view.

3. E. Snsaroli, A. Widom and Y. Srivastava, "Dynamical Casimir Effect and (1/ $\omega)$-noise", Invited Talk by Y. Srivastava at the Chia Conference on the Interface Between Condensed Matter and High Energy Physics, September 1992. ( To appear in Nuclear Physics ).

4. Y. Srivastava, A. Widom, M. Friedman and O. Panella, "Compact QED on the Lattice and the Coulomb Potential", Physics Letters B 298, 405 (1993).

5. Y. Srivastava and A. Widom, "Interference Between Past and Future Events in $\phi \rightarrow K \bar{K}$ Decays", LNF Frascati Preprint, February 1993.

6. "N-Particle Amplitudes for Large N", in Particles, Fields and Cosmology, P. Nath and S. Reucroft (eds.), World Scientific (Singapore 1992)

7. "Classical $\lambda \phi^{4}$ Theory in $3+1$ Dirnensions" (with H. Goldberg and D. Nash), Phys. Rev. D46, 2585 (1992).

8. "Exact Nonperturbative Unitary Amplitudes for $1 \rightarrow 8$ Transitions in a Fieid Theoretic Model" (with H. Goldberg), Fhys.Lett. B291, 125 (1992).

III. PERSONNEL SUPPORTED BY THIS GRANT

1. Y. N. Srivastava (Co-Principal Investigator)

2. M. T. Vaughn (Co-Principal Investigator)

3. Orlando Panella (Graduate Research Assistant

4. Vladan Lucic (Graduate Research Assistant

5. Elisabetta Sassaroli (Graduate Research Assistant 

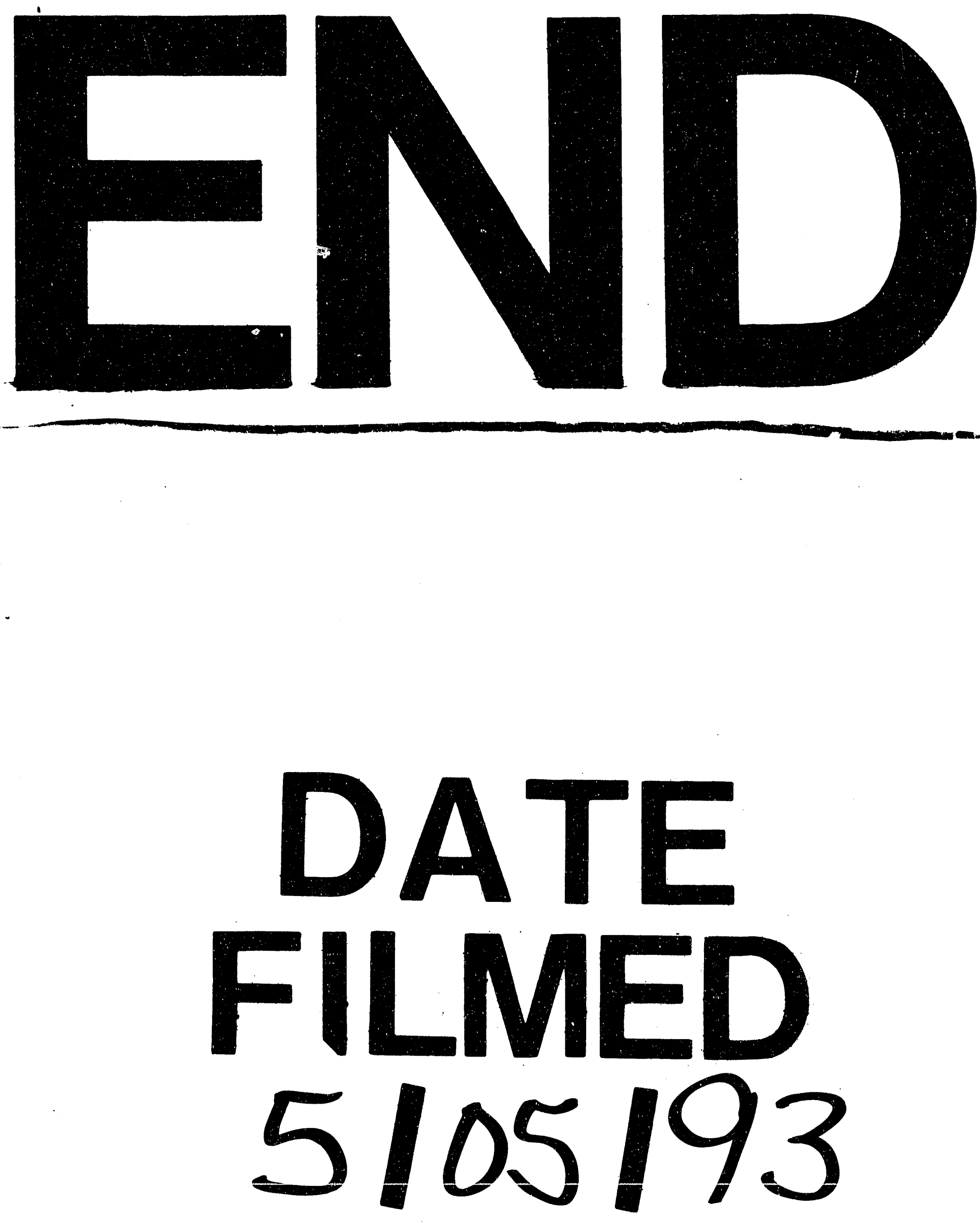
